TRANSACTIONS OF THE

AMERICAN MATHEMATICAL SOCIETY

Volume 358, Number 1, Pages 11-37

S 0002-9947(05)04085-7

Article electronically published on August 25, 2005

\title{
POLAR SETS ON METRIC SPACES
}

\author{
JUHA KINNUNEN AND NAGESWARI SHANMUGALINGAM
}

\begin{abstract}
We show that if $X$ is a proper metric measure space equipped with a doubling measure supporting a Poincaré inequality, then subsets of $X$ with zero $p$-capacity are precisely the $p$-polar sets; that is, a relatively compact subset of a domain in $X$ is of zero $p$-capacity if and only if there exists a $p$-superharmonic function whose set of singularities contains the given set. In addition, we prove that if $X$ is a $p$-hyperbolic metric space, then the $p$-superharmonic function can be required to be $p$-superharmonic on the entire space $X$. We also study the the following question: If a set is of zero $p$ capacity, does there exist a $p$-superharmonic function whose set of singularities is precisely the given set?
\end{abstract}

\section{INTRODUCTION}

Sets of zero capacity in potential theory play the same role as sets of zero measure in the study of $L^{p}$-spaces. Given a metric measure space $X$, functions in the equivalence classes in the Sobolev space $N^{1, p}(X)$ agree up to sets of zero $p$-capacity. The purpose of this note is to characterize sets of zero $p$-capacity in terms of the singular sets of $p$-superharmonic functions.

A polar set of a metric space is a set $E \subset X$ so that there exists a $p$-superharmonic function which takes on the value of infinity at every point in $E$. When $X$ is a Euclidean space and $E$ is a subset of a domain in this Euclidean space with zero $p$-capacity, then there exists a $p$-superharmonic function on the domain taking on the value of infinity at each point of $E$. On the other hand, it is known that polar sets have zero $p$-capacity. Hence, the collection of all sets of zero $p$-capacity in $X$ and the collection of all polar sets in $X$ are one and the same collection (see [HKM] and the references therein). We extend this result to the setting of metric measure spaces that are equipped with a doubling measure and supporting a $(1, q)$ Poincaré inequality for some $q$ with $1 \leq q<p$. For recent results on analysis and potential theory on metric spaces we refer to [B], BMS], C], KaS], KKM], KinM1, KinM2, KSh, KoM], KST], Sh1-Sh3. A very nice general overview of the theory can be found in $[\mathrm{He}$.

It should be noted here that unlike in the setting of Riemannian manifolds, in general metric measure spaces $p$-superharmonic functions need not be associated in any way with a corresponding differential equation. Hence the technique of sweeping of measures developed in Kil1 and Kil2 is not applicable in the generality considered in this paper. However, we do follow the general idea of the proof given

Received by the editors February 27, 2003.

2000 Mathematics Subject Classification. Primary 31C45, 49N60.

Key words and phrases. Minimizers, variational integrals, polar sets, zero capacity sets.

(C)2005 American Mathematical Society Reverts to public domain 28 years from publication 
in Kil1 in proving the main result of this paper, Proposition 3.2. Proposition 3.2, which states that zero $p$-capacity sets are $p$-polar, together with Proposition 2.2, which states that $p$-polar sets have zero $p$-capacity, give a characterization of zero $p$-capacity sets in terms of $p$-polar sets. Observe that the $p$-superharmonic functions which identify a closed $p$-polar set $E$ also act as a barrier function for the points in $E$, considered as boundary points of the open set $\Omega \backslash E$ whenever $\Omega$ is a domain containing $E$. Thus the study of zero $p$-capacity sets in terms of $p$-polar sets is of interest in potential theory.

This note is organized as follows. The first section gathers together the necessary background such as the definition of Newtonian (Sobolev) spaces, capacity, and superharmonic functions. In the second section we prove that $p$-polar sets are of zero $p$-capacity. The third section is focused toward proving that zero $p$-capacity sets are $p$-polar by using the tools developed in KinM2 and the general idea of the proof from [Kil1]. It is also interesting to know when is it true that given a set of zero $p$-capacity there is a function that is $p$-superharmonic on the whole space $X$ so that the given set is a subset of the singularity set of a global superharmonic function. In the fourth section we give a partial answer to this question by demonstrating that whenever $X$ is a $p$-hyperbolic metric space, such global $p$-superharmonic functions can be constructed. It is also interesting to know whether a zero $p$-capacity set is precisely the set of all singularities of a given $p$-superharmonic function. Sections 5 through 7 are devoted to answering this question under a modification of what is meant by a $p$-harmonic function using the approach of [C]. The method is based and potential estimates originally developed in KilM1 and [KilM2 (see also Mi]). On metric spaces potential estimates have been studied in [BMS.

\section{Preliminaries}

We assume that $(X, d, \mu)$ is a metric measure space endowed with a metric $d$ and a non-trivial Borel-regular measure $\mu$. We assume in addition that $X$ is connected and proper, that is, closed and bounded subsets of $X$ are compact. While the results in this note remain valid if we assume that $X$ is locally compact rather than proper, we assume properness for the sake of simplifying notation. The proof of the results in this note can be extended to the locally compact case with some mild modifications.

In this section we recall basic definitions and describe the general setup of our study.

1.1. Upper gradients. Let $u$ be a real-valued function on $X$. A non-negative Borel measurable function $g: X \rightarrow[0, \infty]$ is said to be an upper gradient of $u$ if for all non-constant compact rectifiable paths $\gamma$ joining points $x$ and $y$ in $X$ we have

$$
|u(x)-u(y)| \leq \int_{\gamma} g d s .
$$

See [C] and [Sh2] for a discussion on upper gradients.

Let $1 \leq p<\infty$. A property is said to hold for $p$-almost all paths, if the set of non-constant paths for which the property fails is of zero $p$-modulus (see, for example, Sh2] for the definition of the $p$-modulus). If (1.2) holds for $p$-almost all paths $\gamma$, then $g$ is said to be a $p$-weak upper gradient of $u$. 
1.3. Newtonian spaces. Let $1 \leq p<\infty$. We define the space $\widetilde{N}^{1, p}(X)$ to be the collection of all functions $u \in L^{p}(X)$ that have a $p$-weak upper gradient $g \in L^{p}(X)$. This space is equipped with a seminorm

$$
\|u\|_{\widetilde{N}^{1, p}(X)}=\|u\|_{L^{p}(X)}+\inf \|g\|_{L^{p}(X)},
$$

where the infimum is taken over all $p$-weak upper gradients of $u$. When $p>1$, by the uniform convexity of $L^{p}(X)$ we have that whenever $u \in N^{1, p}(X)$ there is a function $g_{u}$ in the $L^{p}(X)$-convex hull formed by the set of all $p$-weak upper gradients of $u$, called the minimal $p$-weak upper gradient of $u$ (or the minimal $p$-integrable $p$-weak upper gradient), so that $g_{u}$ is a $p$-weak upper gradient of $u$ and

$$
\left\|g_{u}\right\|_{L^{p}(X)}=\inf \|g\|_{L^{p}(X)},
$$

where the infimum is over all $p$-weak upper gradients $g$ of $u$; see [C] or [Sh1]. Indeed, in this case, if $g$ is another $p$-integrable $p$-weak upper gradient of $u$, then $g_{u} \leq g$ almost everywhere in $X$.

We define an equivalence relation in $\widetilde{N}^{1, p}(X)$ by saying that $u \sim v$ if

$$
\|u-v\|_{\widetilde{N}^{1, p}(X)}=0 .
$$

The Newtonian space $N^{1, p}(X)$ is defined to be the space $\widetilde{N}^{1, p}(X) / \sim$ with the norm

$$
\|u\|_{N^{1, p}(X)}=\|u\|_{\widetilde{N}^{1, p}(X)} .
$$

For basic properties of the Newtonian spaces we refer to Sh2]. We recall here some facts for future reference. It can be shown that $N^{1, p}(X)$ is a Banach space. The functions in $N^{1, p}(X)$ are absolutely continuous on $p$-almost every path, which means that $u \circ \gamma$ is absolutely continuous on $[0, \operatorname{length}(\gamma)]$ for $p$-almost every rectifiable arc-length parametrized path $\gamma$ in $X$.

The $p$-capacity of a set $E \subset X$ is defined by

$$
\operatorname{cap}_{p}(E)=\inf _{u}\|u\|_{N^{1, p}(X)}^{p},
$$

where the infimum is taken over all functions $u \in N^{1, p}(X)$, whose restriction to $E$ is bounded below by 1 . A property is said to hold $p$-quasieverywhere, if it holds except on a set of $p$-capacity zero. Capacity is the natural measure for exceptional sets of Sobolev functions. It is easy to see that sets of zero capacity are also of measure zero, but the converse is not true in general. See KinM1 for more properties of the capacity.

Let $E$ be an arbitrary subset of $X$. Following [KKM] we define $N_{0}^{1, p}(E)$ to be the set of functions $u \in N^{1, p}(X)$ for which

$$
\operatorname{cap}_{p}(\{x \in X \backslash E: u(x) \neq 0\})=0 .
$$

The space $N_{0}^{1, p}(E)$ equipped with the norm

$$
\|u\|_{N_{0}^{1, p}(E)}=\|u\|_{N^{1, p}(X)}
$$

is the Newtonian space with zero boundary values. The norm is unambiguously defined by [Sh1] and the space $N_{0}^{1, p}(E)$ with this norm is a Banach space. 
1.4. Poincaré inequalities. A metric measure space $X$ is said to be doubling if there is a constant $c_{d} \geq 1$ so that

$$
\mu(B(z, 2 r)) \leq c_{d} \mu(B(z, r))
$$

for every open ball $B(z, r)$ in $X$. The constant $c_{d}$ in (1.5) is called the doubling constant of $\mu$. Note that by the doubling property, if $B(y, R)$ is a ball in $X$, $z \in B(y, R)$ and $0<r \leq R<\infty$, then

$$
\frac{\mu(B(z, r))}{\mu(B(y, R))} \geq c\left(\frac{r}{R}\right)^{Q}
$$

for some $c$ and $Q$ depending only on the doubling constant. Let $1 \leq q<\infty$. In the following discussion, whenever $A \subset X$ is a measurable set, $u_{A}$ denotes the quantity

$$
\frac{1}{\mu(A)} \int_{A} u d \mu=f_{A} u d \mu \text {. }
$$

The space $X$ is said to support a weak $(1, q)$-Poincaré inequality if there are constants $c_{0}>0$ and $\tau \geq 1$ such that

$$
f_{B(z, r)}\left|u-u_{B(z, r)}\right| d \mu \leq c_{0} r\left(f_{B(z, \tau r)} g^{q} d \mu\right)^{1 / q}
$$

for all balls $B(z, r)$ in $X$, for all integrable functions $u$ in $B(z, r)$ and for all upper gradients $g$ of $u$. If $\tau=1$, then $X$ is said to support a (strong) $(1, q)$-Poincaré inequality.

A result of $[\mathrm{HaK}$ ] shows that in a doubling measure space a weak $(1, q)$-Poincaré inequality implies a weak $(t, q)$-Poincaré inequality for some $t>q$ possibly with possibly different constants $\tau$ and $c_{0}$. More precisely, there exist constants $c>0$ and $\tau^{\prime} \geq 1$ such that

$$
\left(f_{B(z, r)}\left|u-u_{B(z, r)}\right|^{t} d \mu\right)^{1 / t} \leq \operatorname{cr}\left(f_{B\left(z, \tau^{\prime} r\right)} g^{q} d \mu\right)^{1 / q}
$$

where $1 \leq t \leq Q q /(Q-q)$ if $q<Q$ and $t \geq 1$ if $q \geq Q$, for all balls $B(z, r)$ in $X$ and for all integrable functions $u$ in $B(z, r)$ and all upper gradients $g$ of $u$. Moreover, in (1.6) and (1.7) we can replace the upper gradient $g$ with any $p$-weak upper gradient in $L_{\text {loc }}^{p}(X)$, because of the result in $[\mathrm{KoM}]$ which states that every $p$-weak upper gradient in $L_{\mathrm{loc}}^{p}(X)$ can be approximated in $L^{p}(X)$ by an upper gradient in $L_{\mathrm{loc}}^{p}(X)$. Indeed, given any $p$-weak upper gradient $g_{1} \in L_{\text {loc }}^{p}(X)$ of $u$ and any $\epsilon>0$ we can find an upper gradient $g_{2}$ so that

$$
\left\|g_{2}-g_{1}\right\|_{L^{p}(X)}<\epsilon .
$$

1.8. Sobolev inequalities. Next we prove a Sobolev-type inequality for functions which vanish on a large set. The paper $[B]$ has a better capacitary version of this inequality, but for our purposes it suffices to consider the more easily proved version below (see [KSh, Lemma 2.1]).

1.9. Lemma. Let $X$ be a doubling metric measure space supporting a weak $(1, q)$ Poincaré inequality for some $1<q<p$. Suppose that $u \in N^{1, p}(X)$, and denote the minimal $p$-weak upper gradient $g_{u}$. Let

$$
A=\{x \in B(z, r):|u(x)|>0\} .
$$


If $\mu(A) \leq \gamma \mu(B(z, r))$ for some $\gamma$ with $0<\gamma<1$, then there is a constant $c>0$ so that

$$
\left(f_{B(z, r)}|u|^{t} d \mu\right)^{1 / t} \leq c r\left(f_{B\left(z, \tau^{\prime} r\right)} g_{u}^{q} d \mu\right)^{1 / q}
$$

where $t$ and $\tau^{\prime}$ are as in (1.7). The constant $c$ depends only on $\gamma$ and the constants $c$ and $\tau^{\prime}$ of (1.7).

We observe that Lemma 1.9 gives a Sobolev inequality for Sobolev functions with zero boundary values. To be more precise, there exists $c>0$ so that for every ball $B(z, r)$ with $0<r \leq \operatorname{diam}(X) / 3$ and for every $u \in N_{0}^{1, p}(B(z, r))$ we have

$$
\left(f_{B(z, r)}|u|^{t} d \mu\right)^{1 / t} \leq c r\left(f_{B(z, r)} g_{u}^{q} d \mu\right)^{1 / q}
$$

This follows easily from Lemma 1.9 after noting that $g_{u}=0$ almost everywhere on $X \backslash B(z, r)$ and by observing that there must be a point on the sphere $\partial B(z, 2 r)$. If there is no such point, then it is easy to construct a function which violates the Poincaré inequality.

1.11. Minimizers. Now we are ready to formulate the minimization problem for the $p$-Dirichlet integral in a metric measure space. By $N_{\text {loc }}^{1, p}(\Omega)$ we mean the space of all functions $u \in L_{\mathrm{loc}}^{p}(\Omega)$ that have an upper gradient in $L_{\mathrm{loc}}^{p}(\Omega)$, where $L_{\mathrm{loc}}^{p}(\Omega)$ is the space of all measurable functions that are $p$-integrable on compact subsets of $\Omega$.

Suppose that $\Omega \subset X$ is open and let $1<p<\infty$. A function $u \in N_{\text {loc }}^{1, p}(\Omega)$ is said to be $p$-harmonic on $\Omega$ if for every relatively compact open subset $\Omega^{\prime}$ of $\Omega$ with $\overline{\Omega^{\prime}} \subset \Omega$ and for every $v \in N^{1, p}\left(\Omega^{\prime}\right)$ with $u-v \in N_{0}^{1, p}\left(\Omega^{\prime}\right)$ we have

$$
\int_{\Omega^{\prime}} g_{u}^{p} d \mu \leq \int_{\Omega^{\prime}} g_{v}^{p} d \mu
$$

where $g_{u}$ and $g_{v}$ are the minimal weak upper gradients of $u$ and $v$, respectively. $p$-harmonic functions on metric measure spaces have been studied in [C], [Sh1], and KSh.

Let $\vartheta \in N^{1, p}(\Omega)$ and let $\psi: \Omega \rightarrow[-\infty, \infty]$ be an arbitrary function. Following KinM2, we define

$$
\mathcal{K}_{\psi, \vartheta}(\Omega)=\left\{v \in N^{1, p}(\Omega): v-\vartheta \in N_{0}^{1, p}(\Omega), v \geq \psi \mu \text {-a.e. in } \Omega\right\} .
$$

A function $u \in \mathcal{K}_{\psi, \vartheta}(\Omega)$ is a solution of the $\mathcal{K}_{\psi, \vartheta}(\Omega)$-obstacle problem with the obstacle $\psi$ and the boundary values $\vartheta$ if

$$
\int_{\Omega} g_{u}^{p} d \mu \leq \int_{\Omega} g_{v}^{p} d \mu
$$

for every $v \in \mathcal{K}_{\psi, \vartheta}(\Omega)$. Here $g_{u}$ and $g_{v}$ are the minimal $p$-weak upper gradients of $u$ and $v$ in $\Omega$, respectively. In other words, the solution of the obstacle problem minimizes the $p$-Dirichlet energy among all functions which lie above the obstacle function $\psi$ and have the boundary values $\vartheta$. If $\psi=-\infty$, then the obstacle does not hinder and a solution of the $\mathcal{K}_{-\infty, \vartheta}(\Omega)$-obstacle problem is said to be a minimizer with boundary values $\vartheta$ in $\Omega$.

We are mostly interested in local properties of solutions of the obstacle problem and especially in defining solutions locally without any requirements on the boundary values. A function $u \in N_{\text {loc }}^{1, p}(\Omega)$ is a $p$-minimizer in $\Omega$ if it is a solution of the $\mathcal{K}_{-\infty, u}\left(\Omega^{\prime}\right)$-obstacle problem for every open set $\Omega^{\prime} \subset \subset \Omega$. On the other hand, a 
function $u \in N_{\text {loc }}^{1, p}(\Omega)$ is a $p$-superminimizer in $\Omega$ if it is a solution of the $\mathcal{K}_{u, u}\left(\Omega^{\prime}\right)$ obstacle problem for every open set $\Omega^{\prime} \subset \subset \Omega$. In the Euclidean case minimizers correspond to solutions and superminimizers correspond to supersolutions of the p-Laplace equation.

It is an easy observation that whenever $\vartheta$ is in $N^{1, p}(X)$, the solution to the $\mathcal{K}_{\psi, \vartheta}$ obstacle problem is also in $N^{1, p}(X)$.

1.12. Definition. A function $u: \Omega \rightarrow(-\infty, \infty]$ is said to be $p$-superharmonic in $\Omega$ if

(i) $u$ is lower semicontinuous and is not identically infinite on any component of $\Omega$, and

(ii) for all relatively compact open subsets $V \subset \subset \Omega$ and for all functions $v \in$ $N^{1, p}(X)$ that are $p$-minimizers in $V$, whenever $v \leq u p$-q.e. in $\Omega \backslash V$ we have $v \leq u$ $p$-q.e. in $V$.

Remark. The second condition above can be replaced with the condition that for all relatively compact subsets $V \subset \subset \Omega$ and for all functions $v \in N^{1, p}(X) \cap \operatorname{Lip}(X)$ that are $p$-minimizers in $V$, whenever $v \leq u p$-q.e. in $\Omega \backslash V$ we have $v \leq u p$ q.e. in $V$, because of the fact that Lipschitz functions are dense in $N^{1, p}(X)$ (see Sh1]) together with [Sh3. If we can approximate general open sets by regular open sets for the Dirichlet problem, this definition coincides with the definition of p-superharmonic functions given in [HKM, Chapter 7].

Definition 1.12 is slightly different from the definition of $p$-superharmonicity given in [KinM2, the difference being in the requirement of comparison with harmonic functions. Let $\Omega$ be an open subset of $X$. Suppose that $V \subset \subset \Omega$ is open and $v \in N^{1, p}(V)$. Let $h_{V}(v)=h(v)$ be the unique continuous $p$-minimizer in $V$ with $v-h(v) \in N_{0}^{1, p}(V)$. Observe that every $p$-minimizer is locally Hölder continuous after redefinition on a set of measure zero by [KSh]. The definition given in [KinM2] replaces requirement (ii) of Definition 1.12 with the condition

(ii') for every relatively compact open set $V \subset \subset \Omega$ and for every function $v \in$ $C(\bar{V}) \cap N^{1, p}(V)$, whenever $v \leq u$ on $\partial V$ we have that $h(v) \leq u$ on $V$.

The object of the next lemma is to prove that the two definitions are indeed equivalent.

1.13. Lemma. A lower semicontinuous function on $\Omega$ that is not identically infinite on any component of $\Omega$ is p-superharmonic if and only if it satisfies condition (ii') above.

Proof. First suppose that $u$ is a $p$-superharmonic function, let $V \subset \subset \Omega$ and let $v \in C(\bar{V}) \cap N^{1, p}(V)$ such that $v \leq u$ on $\partial V$. For each $\epsilon>0$ there is a compactly supported Lipschitz function $\varphi_{\epsilon}$ on $X$ so that $\left|v-\varphi_{\epsilon}\right| \leq \epsilon$ on $\partial V$. Note that $\varphi_{\epsilon} \in N^{1, p}(X)$. Since

$$
\min \left\{\varphi_{\epsilon}+\epsilon-v, 0\right\} \in N_{0}^{1, p}(V)
$$

and consequently

$$
\min \left\{h\left(\varphi_{\epsilon}\right)+\epsilon-h(v), 0\right\} \in N_{0}^{1, p}(V),
$$

we have by the comparison theorem (see Lemma 3.4 in [KinM2] that

$$
h\left(\varphi_{\epsilon}\right)-\epsilon \leq h(v) \leq h\left(\varphi_{\epsilon}\right)+\epsilon
$$


on $V$. We observe that the function

$$
h= \begin{cases}h\left(\varphi_{\epsilon}\right) & \text { on } V, \\ \varphi_{\epsilon} & \text { on } X \backslash V,\end{cases}
$$

belongs to $N^{1, p}(X)$. Moreover, since $u \geq v \geq \varphi_{\epsilon}-\epsilon$ on $\partial V$ and $u$ is $p$-superharmonic, we have $u \geq h\left(\varphi_{\epsilon}\right)-\epsilon$ on $V$. Hence $u \geq h(v)-2 \epsilon$ on $V$. Letting $\epsilon \rightarrow 0$ we see that $u \geq h(v)$ on $V$.

Now suppose that $u$ satisfies the condition (ii'). Then $u$ is a $p$-superharmonic function in the sense of [KinM2, and hence by [KinM2, Corollary 7.8] the functions

$$
u_{i}=\min \{u, i\}, \quad i=1,2, \ldots,
$$

are superminimizers on $\Omega$. Let $V \subset \subset \Omega$ and let $v \in N^{1, p}(X)$ so that $v$ is a $p$ minimizer on $V$ and $v \leq u$ p-q.e. on $\Omega \backslash V$. By [Sh1, Lemma 4.9] the sequence $v_{i}=\min \{v, i\}$ is in $N^{1, p}(X)$ and $v_{i} \rightarrow v$ in $N^{1, p}(X)$. Thus by [Sh3, Theorem 1.1], $h\left(v_{i}\right)$ converges locally uniformly to $v=h(v)$ in $V$. Since $v \leq u p$-q.e. on $\partial V$, we have $v_{i} \leq u_{i}$ p-q.e. on $\partial V$. From this it follows that $h\left(v_{i}\right) \leq u_{i} \leq u$ on $V$; see the discussion following the proof of Corollary 4.8 in [Sh3]. Letting $i \rightarrow \infty$, we see that $h(v) \leq u$ on $V$, and hence $u$ is a $p$-superharmonic function on $\Omega$.

1.14. General assumptions. From now on we assume without further notice that the metric measure space $X$ is connected, proper, and is equipped with a doubling Borel regular measure for which the measure of every open set is positive and that the measure of every bounded set is finite. Furthermore we assume that the space supports a weak $(1, q)$-Poincaré inequality for some $q$ with $1<q<p$. We assume throughout this paper that $\Omega$ is a bounded open set in $X$ with the property that $\operatorname{cap}_{p}(X \backslash \Omega)>0$.

\section{Polar Sets are Zero CAPACITY SETS}

Let $\Omega \subset X$ be open.

2.1. Definition. A set $E \subset \Omega$ is said to be $p$-polar if there is a $p$-superharmonic function in $\Omega$ so that $u=\infty$ on $E$.

2.2. Proposition. Let $u$ be a p-superharmonic function on $\Omega$, and let

$$
E=\{x \in \Omega: u(x)=\infty\} .
$$

Then E has zero p-capacity.

Proof. Let $F$ be any compact subset of $E$. It suffices to show that the $p$-capacity of $F$ is zero.

Suppose that the $p$-capacity of $F$ is positive. Let $B$ be a relatively compact subdomain of $\Omega$ that contains $F$, and let $v$ be the $p$-potential of the condenser $(F ; B)$; that is, $v=1$ on $F, v=0$ on $X \backslash B, 0 \leq v \leq 1, v$ is $p$-minimizing on $B \backslash F$, and $v$ is in $N^{1, p}(X)$. Since $p$-superharmonic functions are lower semicontinuous and hence are locally bounded from below, we may assume that $u$ is non-negative on $B$. As $B \backslash F$ is a relatively compact open subset of $\Omega$ and $k v \leq u$ on $\Omega \backslash(B \backslash F)$ for every $k=1,2, \ldots$, and $u$ is a $p$-superharmonic function, we have $k v \leq u$ on $B \backslash F$ for every $k=1,2, \ldots$. We can choose $B$ so that there is a point $y \in B$ with $u(y)$ finite. Then $y$ is in $B \backslash F$. Hence

$$
0 \leq k v(y) \leq u(y)<\infty
$$

for every positive $k$ and $v(y)=0$. 
As the $p$-capacity of $F$ is positive, $u$ is a non-constant $p$-minimizing function on $B \backslash F$, and hence by the strong comparison theorem, $v(y)>0$, thus providing the contradiction.

2.3. Definition. A function $f: \Omega \rightarrow[-\infty, \infty]$ is $p$-quasicontinuous if for every $\epsilon>0$ there exists an open set $E$ such that $\operatorname{cap}_{p}(E)<\epsilon$ and the restriction of $f$ to $\Omega \backslash E$ is continuous.

It should be noted that functions in $N^{1, p}(X)$ and $N_{\text {loc }}^{1, p}(X)$ are $p$-quasicontinuous; see Remark 4.4 of [Sh2].

2.4. Corollary. Let $u$ be a p-superharmonic function on $\Omega$. Then $u$ is $p$-quasicontinuous.

Proof. By [KinM2, Corollary 7.8] the functions

$$
u_{i}=\min \{u, i\}, \quad i=1,2, \ldots,
$$

are $p$-superminimizers in $\Omega$, and in particular, they belong to $N_{\text {loc }}^{1, p}(\Omega)$. Hence the functions $u_{i}$ are $p$-quasicontinuous, and the result follows from Proposition 2.2 and the fact that the $p$-capacity is a Choquet capacity. Indeed, the sets

$$
U_{i}=\{x \in \Omega: u(x)>i\}
$$

are open and $\operatorname{cap}_{p}\left(U_{i}\right) \rightarrow 0$ as $i \rightarrow \infty$.

\section{ZERO CAPACITY SETS ARE POLAR SETS}

3.1. Lemma. Let $E \subset \subset \Omega$ such that $\operatorname{cap}_{p}(E)=0$. Then there exists a sequence of functions $\varphi_{i} \in N^{1, p}(X)$ so that $\varphi_{i}=0$ on $X \backslash \Omega, 0 \leq \varphi_{i} \leq 1, \varphi_{i}=1$ on $E, \varphi_{i}$ is a p-superharmonic p-superminimizer on $\Omega, \varphi_{i} \rightarrow 0$ in $N^{1, p}(X)$, and $\varphi_{i}$ monotonically decreases to zero p-q.e. in $\Omega$.

Proof. Since $\operatorname{cap}_{p}(E)=0$, there exists a sequence of open sets $U_{i} \subset \subset \Omega$ with $E \subset U_{i} \subset U_{i-1}$ so that $\operatorname{cap}_{p}\left(U_{i}\right) \leq 2^{-i}$. Let $\widetilde{\varphi}_{i}$ be the $p$-potential of the condenser $\left(U_{i} ; \Omega\right)$, and let $\varphi_{i}$ be the lower semicontinuous regularization of $\widetilde{\varphi}_{i}$ given by KinM2, Proposition 7.4]. The functions $\varphi_{i}$ satisfy the conditions of the lemma.

3.2. Proposition. Let $\Omega \subset X$ be a bounded open set, and let $\psi_{i} \in N^{1, p}(X)$ be a p-q.e. monotonic decreasing sequence of non-negative functions so that $\psi_{i} \rightarrow \psi$ in $N^{1, p}(X)$. Let $u_{i}$ be the solution to the $\mathcal{K}_{\psi_{i}, \psi_{i}}(\Omega)$-obstacle problem. Then there exists a function $u \in N^{1, p}(X)$ so that $u_{i}$ monotonically decreases $p$-q.e. in $\Omega$ to $u$ and $u$ is the solution to the $\mathcal{K}_{\psi, \psi}(\Omega)$-obstacle problem.

The above proposition is a complementary result to KinM2, Theorem 6.1]. To prove this proposition we need the following lemma. This lemma is from KinM2, Lemma 3.5].

3.3. Lemma. Let $\psi \in N^{1, p}(X)$ be a non-negative function and let $v$ be a solution to the $\mathcal{K}_{\psi, \psi}(\Omega)$-obstacle problem. If $u \in N^{1, p}(X)$ is a $p$-superminimizer on $\Omega$ with $\min \{u, v\} \in \mathcal{K}_{\psi, \psi}(\Omega)$, then $u \geq v$ p-q.e. on $\Omega$. Moreover, if $\widetilde{u}$ and $\widetilde{v}$ are the lower semicontinuous regularizations of $u$ and $v$ respectively, we have $\widetilde{u} \geq \widetilde{v}$ everywhere on $\Omega$. 
Proof. For the proof of the first part, see [KinM2, Lemma 3.5]. While their proof involves comparing $u$ and $v$ outside of a relatively compact subdomain of $\Omega$, as $u$ and $v$ are in $N^{1, p}(X)$, the relative compact open subsets are unnecessary. Moreover, by [KinM2, Theorem 7.14] we have

$$
\widetilde{v}(x)=\operatorname{ess} \liminf _{y \rightarrow x} \widetilde{v}(y) \leq \operatorname{ess} \liminf _{y \rightarrow x} \widetilde{u}(y)=\widetilde{u}(x)
$$

for every $x \in \Omega$.

Now we are ready to prove Proposition 3.2.

Proof of Proposition 3.2. Since $u_{i} \geq \psi_{i} \geq \psi_{i+1}$, therefore $u_{i} \in \mathcal{K}_{\psi_{i+1}, \psi_{i}}(\Omega)$. As $u_{i}$ is a $p$-superminimizer on $\Omega$ and $u_{i+1}$ is the solution to the obstacle problem with obstacle $\psi_{i+1}$ and boundary data $\psi_{i+1} \leq \psi_{i}$, by Lemma 3.3 above we have $u_{i} \geq$ $u_{i+1}$. Without loss of generality we may assume that $u_{i}$ is lower semicontinuous in $\Omega$; see [KinM2, Theorem 5.1]. Hence $\left(u_{i}\right)_{i}$ is a monotone decreasing sequence. Let

$$
u=\lim _{i \rightarrow \infty} u_{n}
$$

As $u_{i} \in L^{p}(\Omega)$ and $u \geq 0$, we have $u \in L^{p}(\Omega)$. Also, as $\psi_{i} \in \mathcal{K}_{\psi_{i}, \psi_{i}}(\Omega)$, we have

$$
\int_{\Omega} g_{u_{i}}^{p} d \mu \leq \int_{\Omega} g_{\psi_{i}}^{p} d \mu
$$

Hence by [KaS, Lemma 3.1] we see that $u \in N^{1, p}(X)$ with

$$
\int_{\Omega} g_{u}^{p} d \mu \leq \liminf _{i \rightarrow \infty} \int_{\Omega} g_{u_{i}}^{p} d \mu \leq \liminf _{i \rightarrow \infty} \int_{\Omega} g_{\psi_{i}}^{p} d \mu \leq \int_{\Omega} g_{\psi}^{p} d \mu .
$$

Since $u_{i} \geq \psi_{i} \geq \psi$, we conclude that $u \geq \psi$ and hence $u \in \mathcal{K}_{\psi, \psi}(\Omega)$ because $u_{i}-\psi_{i} \in N_{0}^{1, p}(\Omega)$ and $\psi_{i}$ converges to $\psi$ in $N^{1, p}(X)$. Let $v$ be the solution to the $\mathcal{K}_{\psi, \psi}(\Omega)$-obstacle problem. Then

$$
\int_{\Omega} g_{v}^{p} d \mu \leq \int_{\Omega} g_{u}^{p} d \mu
$$

Also by Lemma 3.3 again, $u_{i} \geq v$, and hence $u \geq v$. Let $\varphi_{i}=\max \left\{v, \psi_{i}\right\}$. Then as $\psi \leq \psi_{i}, \varphi_{i} \in \mathcal{K}_{\psi_{i}, \psi_{i}}(\Omega)$, and hence

$$
\int_{\Omega} g_{u_{i}}^{p} d \mu \leq \int_{\Omega} g_{\varphi_{i}}^{p} d \mu .
$$

As $\psi_{i}$ monotonically decreases to $\psi$ p-q.e. in $\Omega$ and $v \geq \psi$, therefore $\varphi_{i} \rightarrow v$ pointwise $p$-q.e. in $\Omega$. Moreover, as $\psi_{i} \rightarrow \psi$ in $N^{1, p}(X)$, we see that $\varphi_{i} \rightarrow v$ in $N^{1, p}(X)$. Therefore,

$$
\liminf _{i \rightarrow \infty} \int_{\Omega} g_{u_{i}}^{p} d \mu \leq \int_{\Omega} g_{v}^{p} d \mu,
$$

and hence,

$$
\int_{\Omega} g_{u}^{p} d \mu \leq \int_{\Omega} g_{v}^{p} d \mu .
$$

Thus we see that

$$
\int_{\Omega} g_{u}^{p} d \mu=\int_{\Omega} g_{v}^{p} d \mu .
$$

By the uniqueness of solutions to obstacle problems (see for example KinM2, Theorem 3.2] or [HKM, Theorem 3.21]) we have $u=v$; that is, $u$ is the solution to the $\mathcal{K}_{\psi, \psi}(\Omega)$-obstacle problem. 
Now we state and prove the main theorem of this section.

3.4. Theorem. Let $x_{0} \in \Omega$ and let $E$ be a relatively compact subset of $\Omega \backslash\left\{x_{0}\right\}$ such that $\operatorname{cap}_{p}(E)=0$. Then there exists a non-negative p-superharmonic function $u$ on $\Omega$ so that $u\left(x_{0}\right)<\infty, u=0$ on $X \backslash \Omega$, and

$$
E \subset\{x \in \Omega: u(x)=\infty\} .
$$

This theorem was proved in the setting of $X=\mathbf{R}^{n}$ by Kilpeläinen; see Kil1, Theorem 4.1]. The proof of this theorem follows the lead of Kil1; however, we avoid their use of Perron-Wiener-Brelot solutions by using Proposition 3.2 instead.

Proof. Let $\varphi_{i}$ be the functions given by Lemma 3.1. There exists an index $i_{1}$ so that $\varphi_{i_{1}} \leq \frac{1}{2}$ in a neighbourhood of $x_{0}$ and $\varphi_{i_{1}}=1$ on a neighbourhood of $E$. Let $\widetilde{v}_{1}$ be the lower semicontinuous regularization of the $p$-superminimizer $\varphi_{i_{1}}$; see KinM2, Theorem 5.1]. Note that as $\varphi_{i_{1}}$ is already a $p$-superharmonic function as well, we have $\widetilde{v}_{1}=\varphi_{i_{1}}$. We inductively construct functions $\widetilde{v}_{n}$ as follows.

Suppose $\widetilde{v}_{1}, \ldots, \widetilde{v}_{n-1}$ have already been chosen so that they are $p$-superharmonic $p$-superminimizers in $\Omega, \widetilde{v}_{i}=0$ on $X \backslash \Omega, \widetilde{v}_{i}=i$ on a neighbourhood of $E$,

$$
\widetilde{v}_{i}\left(x_{0}\right) \leq \sum_{k=1}^{i} 2^{-k},
$$

and $\widetilde{v}_{i} \in N^{1, p}(X)$.

Let $v_{n, j}$ be the solution to the obstacle problem on $\Omega$ with obstacle $\widetilde{v}_{n-1}+\varphi_{j}$ and boundary data 0 . Since $\varphi_{j} \rightarrow 0$ in $N^{1, p}(X)$ and $\varphi_{j}$ monotonically decreases to $0 p$-q.e., we see that $\widetilde{v}_{n-1}+\varphi_{j}$ monotonically decreases to $\widetilde{v}_{n-1} p$-q.e. and in $N^{1, p}(X)$. Now by Proposition $3.2, v_{n, j}$ monotonically decreases to $\widetilde{v}_{n-1} p$-q.e., and hence its lower semicontinuous regularization monotonically decreases to $\widetilde{v}_{n-1}$ everywhere in $\Omega$; see KinM2, Theorem 5.1]. Hence there is an index $i_{n}$ so that the lower semicontinuous regularization of $v_{n, i_{n}}$ is bounded by $\sum_{k=1}^{n} 2^{-k}$ at $x_{0}$, and as $v_{n, i_{n}}=n$ on a neighbourhood of $E$, the lower semicontinuous regularization of $v_{n, i_{n}}$ also takes on the value of $n$ in a neighbourhood of $E$. Set $\widetilde{v}_{n}$ to be this lower semicontinuous regularization of $v_{n, i_{n}}$.

The sequence $\left(\widetilde{v}_{n}\right)$ has the following properties:

- $\widetilde{v}_{n} \geq 0$ and is $p$-superharmonic in $\Omega$,

- $\widetilde{v}_{n}=0$ on $X \backslash \Omega$,

- $\widetilde{v}_{n} \in N^{1, p}(X)$,

- $\widetilde{v}_{n}=n$ on $E$,

- $\widetilde{v}_{n+1} \geq \widetilde{v}_{n}$ pointwise on $\Omega$, and

- $\widetilde{v}_{n}\left(x_{0}\right) \leq \sum_{k=1}^{n} 2^{-k}$.

Let $u=\lim _{n \rightarrow \infty} \widetilde{v}_{n}$. By [KinM2, Lemma 7.1] and by Lemma 1.13 above, since

$$
u\left(x_{0}\right) \leq \sum_{k=1}^{\infty} 2^{-k}
$$

is finite, $u$ is $p$-superharmonic on $\Omega$. Moreover, it is easy to see that $u=\infty$ on E.

Remark. In the Euclidean setting, in the proof of Lemma 3.1 we can approximate $\Omega$ by regular open sets $\Omega_{j} \subset \Omega$ and the open sets $U_{j}$ by regular open sets $\widetilde{U}_{j}$; this technique has been used extensively in [HKM] to study the Perron-Wiener-Brelot 
solutions. In this case, we can ensure that the functions $\varphi_{i}$ are all continuous, and then it follows from [KinM2, Theorem 5.5] that the functions $\widetilde{v}_{i}$ constructed in the above proof are all continuous. Unfortunately, in the general setting of metric spaces considered in this note, such approximations are not known to be possible.

\section{The EXISTENCE of GLOBAL $p$-SUPERHARMONIC MAPS DEFINING $p$-POLAR SETS IN A $p$-HYPERBOLIC METRIC SPACE}

Following [ $[\mathrm{Ho}$, we say that a metric space is $p$-hyperbolic if there exists a compact set $C \subset X$ so that the relative capacity $\operatorname{Cap}_{p}(C: X)$ is positive. Here

$$
\operatorname{Cap}_{p}(C: X)=\lim _{i \rightarrow \infty} \operatorname{Cap}_{p}\left(C: \Omega_{i}\right)
$$

where $\left\{\Omega_{i}\right\}_{i}$ is an increasing sequence of bounded domains in $X$ such that $X=$ $\bigcup_{i} \Omega_{i}$. It is easily seen that a metric space is $p$-hyperbolic if and only if it is not possible to find a sequence of compactly supported functions $f_{i} \in N^{1, p}(X)$ so that $f_{i} \rightarrow 1$ in $N_{\text {loc }}^{1, p}(X)$, while their energies

$$
\int_{X} g_{f_{i}}^{p} d \mu
$$

remain bounded.

4.1. Theorem. If $X$ is a p-hyperbolic metric measure space equipped with a doubling measure supporting a $(1, q)$-Poincaré inequality for some $1 \leq q<p$, and if $E \subset X$ is a compact set so that $\operatorname{cap}_{p}(E)=0$, then there exists a non-negative function $u$ on $X$ so that $u$ is p-superharmonic in $X$ and

$$
E \subset\{x \in X: u(x)=\infty\} .
$$

The proof of this theorem follows along the same lines as the proof of Theorem 3.4, once an analog of Lemma 3.1 and Proposition 3.2 are proven in the setting $\Omega=X$.

4.2. Definition. In what follows, the family $N_{\text {loc }, 0}^{1, p}(X)$ denotes the collection of all functions $f$ on $X$ for which there exists a sequence of compactly supported functions $f_{i} \in N^{1, p}(X)$ so that $f_{i} \rightarrow f$ in $N_{\text {loc }}^{1, p}(X)$ and the corresponding sequence of energies

$$
\int_{X} g_{f_{i}}^{p} d \mu
$$

is bounded independent of $i$. Weak limits of the functions $g_{f_{i}}$ can be easily seen to be $p$-weak upper gradients of $f$ in $X$. From the fact that $f_{i} \rightarrow f$ in $N_{\text {loc }}^{1, p}(X)$ we can infer that $g_{f_{i}} \rightarrow g_{f}$ in $L^{p}(X)$ and that $g_{f} \in L^{p}(X)$.

It is easy to see that if $\lambda>0$ and $f, h \in N_{\text {loc }, 0}^{1, p}(X)$, then the functions $\min \{\lambda, f\}$ and $\max \{f, h\}$ are in $N_{\text {loc }, 0}^{1, p}(X)$. Furthermore, $N_{\text {loc }, 0}^{1, p}(X)$ is a vector space endowed with a family of seminorms inherited from $N_{\text {loc }}^{1, p}(X)$.

4.3. Definition. Let $f \in N_{\text {loc }}^{1, p}(X)$, and let $\mathcal{K}_{f}$ denote the collection of all functions $u \in N_{\text {loc }}^{1, p}(X)$ such that $u-f \in N_{\text {loc }, 0}^{1, p}(X)$ and $u \geq f p$-q.e. in $X$. By a solution to the obstacle problem $\mathcal{K}_{f}$ we mean a function $u \in \mathcal{K}_{f}$ so that

$$
\int_{X} g_{u}^{p} d \mu \leq \int_{X} g_{v}^{p} d \mu
$$


whenever $v \in \mathcal{K}_{f}$. Observe that

$$
\int_{X} g_{u}^{p} d \mu \leq \int_{X} g_{f}^{p} d \mu .
$$

4.4. Lemma. Let $X$ be a p-hyperbolic metric space. Given $E \subset X$ so that $\operatorname{cap}_{p}(E)=0$, there exists a sequence of functions $\varphi_{i} \in N_{\text {loc }, 0}^{1, p}(X)$ so that $\varphi_{i}=1$ on a neighbourhood of $E, \varphi_{i}$ is a p-superharmonic p-superminimizer on $X$,

$$
\int_{X} g_{\varphi_{i}}^{p} d \mu \rightarrow 0
$$

as $i \rightarrow \infty$, and $\varphi_{i}$ monotonically decreases to 0 pointwise p-q.e. in $X$.

The proof of Lemma 4.4 depends on the following lemma.

4.5. Lemma. Let $X$ be a p-hyperbolic space equipped with a doubling measure supporting a $(1, p)$-Poincaré inequality. Given a bounded function $f \in N_{\text {loc }}^{1, p}(X)$ with finite energy, there is one, and only one, solution to the obstacle problem $\mathcal{K}_{f}$ in the class $N_{\mathrm{loc}, 0}^{1, p}(X)$. Such a solution is p-superharmonic and is a $p$-superminimizer in $X$.

Proof. We denote the bound of $f$ by $M$. Let $\Omega_{i}$ be an increasing sequence of bounded domains in $X$ so that

$$
X=\bigcup_{i=1}^{\infty} \Omega_{i}
$$

As $\bar{\Omega}_{i}$ is a compact set and $N_{\text {loc }, 0}^{1, p}(X) \subset N_{\text {loc }}^{1, p}(X)$, we see that the solution $u_{i}$ to the obstacle problem $\mathcal{K}_{f, f}\left(\Omega_{i}\right)$ exists and lies in $N_{\text {loc }, 0}^{1, p}(X)$. By Lemma 3.3 we see that $f \leq u_{i} \leq u_{i+1} \leq M$ p-q.e. on $X$.

On the other hand, by the energy minimization property of $u_{i}$, we have

$$
\int_{X} g_{u_{i}}^{p} d \mu=\int_{\Omega_{i}} g_{u_{i}}^{p} d \mu+\int_{X \backslash \Omega_{i}} g_{f}^{p} d \mu \leq \int_{X} g_{f}^{p} d \mu<\infty,
$$

where we know that the last integral in the above chain of inequalities is finite by assumption. Hence the sequence $u_{i}$ has the property that

$$
\int_{X} g_{u_{i}}^{p} d \mu
$$

is bounded independent of $i$. Thus the limit function $u=\lim _{i \rightarrow \infty} u_{i}$ lies in the collection $N_{\text {loc }}^{1, p}(X)$. Observe that $u_{i} \in N_{\text {loc }}^{1, p}(X)$ and that $\left\|u_{i}\right\|_{N^{1, p}\left(\Omega_{k}\right)}$ are bounded above by a constant independent of $i$. Therefore, as

$$
u-f=\lim _{i \rightarrow \infty}\left(u_{i}-f\right)
$$

with $u_{i}-f$ of compact support, we see that $u \in \mathcal{K}_{f}$.

Suppose $v \in \mathcal{K}_{f}$. Then as $v \geq f$, there exists a sequence of non-negative functions $w_{i} \in N^{1, p}(X)$ with compact support so that

$$
v=\lim _{i \rightarrow \infty}\left(w_{i}+f\right)
$$

and (because of Mazur's lemma) so that

$$
\int_{X} g_{v}^{p} d \mu=\lim _{i \rightarrow \infty} \int_{X} g_{w_{i}+f}^{p} d \mu .
$$


Fix $\epsilon>0$ and choose $w_{i}$ so that

$$
\int_{X} g_{v}^{p} d \mu \geq \int_{X} g_{w_{i}+f}^{p} d \mu-\epsilon .
$$

Since

$$
X=\bigcup_{j=1}^{\infty} \Omega_{j}
$$

we can find $\Omega_{j}$ so that the compact support of $w_{i}$ is contained in $\Omega_{j}$; thus $w_{i}+f \in$ $\mathcal{K}_{f, f}\left(\Omega_{j}\right)$ for sufficiently large $j$. Now by the minimal energy property of $u_{j}$, we see that

$$
\int_{X} g_{u_{j}}^{p} d \mu \leq \int_{X} g_{w_{i}+f}^{p} d \mu .
$$

Hence for sufficiently large $j$ we have that

$$
\int_{X} g_{u_{j}}^{p} d \mu \leq \int_{X} g_{v}^{p} d \mu+\epsilon .
$$

Letting $j \rightarrow \infty$ and then $\epsilon \rightarrow 0$, we see that

$$
\int_{X} g_{u}^{p} d \mu \leq \int_{X} g_{v}^{p} d \mu
$$

that is, $u$ is a solution to the obstacle problem $\mathcal{K}_{f}$.

Since the monotone increasing sequence of functions $\left(u_{i}\right)_{i \geq j}$ are $p$-superharmonic and are $p$-superminimizers in $\Omega_{j}$, by KinM2 we see that $u$ is itself such a function on $\Omega_{j}$ for every $j$; that is, $u$ is $p$-superharmonic and is a $p$-superminimizer in $X$.

It now remains to show that no other solution to the obstacle problem exists. Suppose $v$ is another solution to the obstacle problem $\mathcal{K}_{f}$. Then as $v \geq f p$-q.e. on $X$, we see by an application of Lemma 3.3 to the domains $\Omega_{j}$ that $v \geq u_{j}$ for each $j$. It follows that $v \geq u p$-q.e. on $X$. As $v \not \equiv u$ and $X$ supports a $(1, p)$-Poincaré inequality (and hence is an $M E C_{p}$-space; see Sh2]), we have $g_{v-u} \not \equiv 0$ or else $v-u$ is a non-zero constant function. By the results in [Sh2], the support of $g_{v-u}$ is contained in the support of $v-u$ itself. As $u \neq \equiv v$, we see that

$$
\mu(\{x \in X: u(x)<v(x)\})>0 .
$$

Note that the set $\{x \in X: u(x)<v(x)\}$ is a countable union of sets of the form

$$
\{x \in X: u(x)<a<v(x)\},
$$

where $a \in \mathbf{R}$ is a positive rational number. Let $a$ be a positive rational number such that

$$
\mu(\{x \in X: u(x)<a<v(x)\})>0
$$

and define

$$
h(x)= \begin{cases}v(x), & \text { if } v(x) \leq a \\ a, & \text { if } u(x)<a<v(x), \\ u(x), & \text { if } u(x) \geq a\end{cases}
$$

It is easily seen that $h \in \mathcal{K}_{f}$, and hence by the minimality of the energy of the solutions,

$$
\int_{X} g_{u}^{p} d \mu=\int_{X} g_{v}^{p} d \mu \leq \int_{X} g_{h}^{p} d \mu=\int_{\{v \leq a\}} g_{v}^{p} d \mu+\int_{\{u \geq a\}} g_{u}^{p} d \mu .
$$


By the above inequality,

$$
\int_{\{v \leq a\}} g_{v}^{p} d \mu=\int_{\{v \leq a\}} g_{u}^{p} d \mu \quad \text { and } \quad \int_{\{u \geq a\}} g_{v}^{p} d \mu=\int_{\{u \geq a\}} g_{u}^{p} d \mu
$$

Hence

$$
\int_{X} g_{h}^{p} d \mu=\int_{X} g_{u}^{p} d \mu-\int_{\{u<a<v\}} g_{u}^{p} d \mu \geq \int_{X} g_{u}^{p} d \mu
$$

which leads us to the conclusion that $g_{u}=0$ almost everywhere in the set

$$
\{x \in X: u(x)<a<v(x)\},
$$

and a similar argument shows that $g_{v}=0$ almost everywhere on this set. Thus $g_{v-u} \leq g_{v}+g_{u}=0$ almost everywhere on the set

$$
\{x \in X: u(x)<a<v(x)\} .
$$

Thus $g_{v-u}=0$ almost everywhere on the set

$$
\{x \in X: u(x)<v(x)\} .
$$

Hence it follows that $u+c \equiv v$ for some constant $c>0$. On the other hand, as $u-f, v-f \in N_{\text {loc }, 0}^{1, p}(X)$ we have $v-u \in N_{\text {loc }, 0}^{1, p}(X)$. Hence as $X$ is $p$-hyperbolic, we have $c=0$, yielding the uniqueness result for the solution to the obstacle problem $\mathcal{K}_{f}$.

Proof of Lemma 4.4. By Lemma 3.1 we obtain a sequence of compactly supported functions $\phi_{i} \in N^{1, p}(X)$ so that $\phi_{i}=1$ on $E$,

$$
\int_{X} g_{\phi_{i}}^{p} d \mu \leq 2^{-i p}
$$

and $\phi_{i}$ monotonically decreases to zero $p$-q.e. in $X$. Let $\varphi_{i}$ be the solution to the $\mathcal{K}_{\phi_{i}}$-obstacle problem constructed in the proof of Lemma 4.5. As $\phi_{i}$ is compactly supported, $\varphi_{i} \in N_{\mathrm{loc}, 0}^{1, p}(X)$ with

$$
\int_{X} g_{\varphi_{i}}^{p} d \mu \leq 2^{-i p}
$$

Moreover, by Lemma 4.5 we see that $\varphi_{i}$ are $p$-superharmonic $p$-superminimizers in $X$.

As $\phi_{i}$ is monotonic decreasing, by construction and by Lemma 3.3 we see that $\left(\varphi_{i}\right)_{i}$ is a monotonic decreasing sequence in $X$. Let $\varphi:=\lim _{i} \varphi_{i}$. Since

$$
\int_{X} g_{\varphi}^{p} d \mu \leq \int_{X} g_{\varphi_{i}}^{p} d \mu \leq 2^{-i p}
$$

we see that $g_{\varphi}=0$ almost everywhere in $X$. As $X$ supports a $(1, p)$-Poincaré inequality (and hence is an $M E C_{p^{-}}$-space; see [Sh2]), we see that $\varphi \geq 0$ is constant $p$-a.e. in $X$. To see that this constant is zero, it suffices to know that $\varphi \in N_{\text {loc }, 0}^{1, p}(X)$.

Indeed, as $\phi_{i}$ has compact support, we see that $\varphi_{i} \in N_{\text {loc }, 0}^{1, p}(X)$. As $\varphi_{i}$ are bounded by 1 , we see that the sequence $\phi_{i, j}$, obtained as the solution to the obstacle problem $\mathcal{K}_{\phi_{i}, \phi_{i}}\left(\Omega_{j}\right)$, is a bounded sequence in $N_{\text {loc }}^{1, p}(X)$. Fix one such relatively compact domain $\Omega_{k}$. Hence by Mazur's lemma we can choose a subsequence of convex combination $\left(\widehat{\phi}_{i, j}\right)_{j}$ of the sequence $\left(\phi_{i, j}\right)_{j}$, so that $\widehat{\phi}_{i, j} \rightarrow \varphi_{i}$ in $N^{1, p}\left(\Omega_{k}\right)$ 
for every relatively compact domain $\Omega_{k}$ in $X$. Fix $\epsilon>0$. Then for every positive integer $i$ we can find a cutoff integer $j_{i}$, so that whenever $j \geq j_{i}$ we have

$$
\left\|\varphi_{i}-\widehat{\phi}_{i, j}\right\|_{N^{1, p}\left(\Omega_{k}\right)}<\epsilon / 2 \text {. }
$$

Similarly, as $\varphi_{i} \rightarrow \varphi$ in a monotonic decreasing fashion and is a bounded sequence in $N^{1, p}\left(\Omega_{k}\right)$, we can obtain a convex combination subsequence

$$
\widetilde{\varphi}_{n}=\sum_{i=n}^{N_{n}} \lambda_{i, n} \varphi_{i}
$$

that converges in $N^{1, p}\left(\Omega_{k}\right)$ to $\varphi$. Thus a corresponding convex combination of the sequence $\widehat{\phi}_{i, j}$ converges in $N^{1, p}\left(\Omega_{k}\right)$ to $\varphi$. More specifically, with $\epsilon>0$ fixed as above, we can find a positive integer $i_{0}$ so that

$$
\left\|\widetilde{\varphi}_{i_{0}}-\varphi\right\|_{N^{1, p}\left(\Omega_{k}\right)}<\epsilon / 2 .
$$

Choosing $j_{0}=\max \left\{j_{i_{0}}, j_{i_{0}+1}, \ldots, j_{N_{i_{0}}}\right\}$ and letting

$$
\widetilde{\phi}_{i_{0}}:=\sum_{i=i_{0}}^{N_{i_{0}}} \lambda_{i, i_{0}} \phi_{i},
$$

it is easy to see that

$$
\left\|\widetilde{\phi}_{i_{0}}-\widetilde{\varphi}_{i_{0}}\right\|_{N^{1, p}\left(\Omega_{k}\right)}<\epsilon / 2 \text {. }
$$

Thus we obtain an approximation $\widetilde{\phi}_{i_{0}}$ of $\varphi$ so that this approximation is compactly supported in $N^{1, p}(X)$, and in addition,

$$
\left\|\widetilde{\phi}_{i_{0}}-\varphi\right\|_{N^{1, p}\left(\Omega_{k}\right)}<\epsilon .
$$

We can now conclude that $\varphi \in N_{\text {loc, } 0}^{1, p}(X)$, and hence cannot be a non-zero constant. Thus $\varphi=0$, and the proof is complete.

Remark. The above proof also shows that if $u_{i} \in N_{\text {loc }, 0}^{1, p}(X)$ is a sequence that converges to a function $u \in N_{\text {loc }}^{1, p}(X)$, with the convergence occurring in $N_{\text {loc }}^{1, p}(X)$, and furthermore, the energies

$$
\int_{X} g_{u_{i}}^{p} d \mu
$$

are bounded independent of $i$, then $u$ is also in $N_{\text {loc }, 0}^{1, p}(X)$. Hence $N_{\text {loc }, 0}^{1, p}(X)$ is a complete space.

4.6. Proposition. Let $\psi_{i} \in N_{\mathrm{loc}, 0}^{1, p}(X)$ be a sequence of $p$-q.e. monotone decreasing sequence of non-negative functions so that $\psi_{i} \rightarrow \psi \in N_{\text {loc }, 0}^{1, p}(X)$, the convergence also occurring in $N^{1, p}(X)$. Let $u_{i}$ be the solution to the obstacle problem $\mathcal{K}_{\psi_{i}}$. Then $u_{i} \rightarrow u$ both monotonically p-q.e. in $X$ and weakly in $N_{\text {loc }}^{1, p}(X)$, where $u$ is the solution to the obstacle problem $\mathcal{K}_{\psi}$.

The proof of Proposition 4.6 is along the same lines as that of Proposition 3.2.

Proof. Lemma 3.3 together with the proof of Lemma 4.5 shows that $u_{i} \geq u_{i+1}$ p-q.e. since $\psi_{i} \geq \psi_{i+1}$. Let

$$
u=\lim _{i \rightarrow \infty} u_{i}
$$


As $u_{i} \geq \psi_{i} \geq \psi$, we see that $u \geq \psi$. Furthermore, as

$$
\int_{X} g_{\psi_{i}}^{p} d \mu
$$

is bounded independent of $i$, we see that so is the sequence of energies

$$
\int_{X} g_{u_{i}}^{p} d \mu
$$

Hence by the remark following the proof of Lemma $4.5, u \in N_{\text {loc, }, 0}^{1, p}(X)$, and thus we can conclude that $u \in \mathcal{K}_{\psi}$. Moreover, as

$$
\int_{X} g_{u_{i}}^{p} d \mu \leq \int_{X} g_{\psi_{i}}^{p} d \mu \rightarrow \int_{X} g_{\psi}^{p} d \mu
$$

we see that

$$
\int_{X} g_{u}^{p} d \mu \leq \int_{X} g_{\psi}^{p} d \mu
$$

Let $v$ denote the solution to the obstacle problem $\mathcal{K}_{\psi}$ (Lemma 4.5 guarantees such a solution). From the fact that $u \in \mathcal{K}_{\psi}$ we see that

$$
\int_{X} g_{u}^{p} d \mu \geq \int_{X} g_{v}^{p} d \mu
$$

By the uniqueness of solutions to obstacle problems (Lemma 4.5), it therefore suffices to show that

$$
\int_{X} g_{u}^{p} d \mu \leq \int_{X} g_{v}^{p} d \mu .
$$

To this end, as in the proof of Proposition 3.2, let $\varphi_{i}=\max \left\{v, \psi_{i}\right\}$. Then $\varphi_{i} \in$ $N_{\text {loc }, 0}^{1, p}(X)$ and $\varphi_{i} \geq \psi_{i}$. Therefore, $\varphi_{i} \in \mathcal{K}_{\psi_{i}}$ and

$$
\int_{X} g_{u_{i}}^{p} d \mu \leq \int_{X} g_{\varphi_{i}}^{p} d \mu
$$

On the other hand, as $\psi_{i} \rightarrow \psi$ in $N^{1, p}(X)$ and $v \geq \psi$, we see that $\varphi_{i} \rightarrow v$ in $N^{1, p}(X)$; that is,

$$
\int_{X} g_{\varphi_{i}}^{p} d \mu \rightarrow \int_{X} g_{v}^{p} d \mu
$$

Therefore

$$
\int_{X} g_{u}^{p} d \mu \leq \liminf _{i \rightarrow \infty} \int_{X} g_{u_{i}}^{p} d \mu \leq \int_{X} g_{v}^{p} d \mu,
$$

completing the proof of the proposition.

Proof of Theorem 4.1. The proof of Theorem 4.1 follows the exact lines as in the proof of Theorem 3.4, with Proposition 4.6 playing the role of Proposition 3.2 and Lemma 3.1, Lemma 4.5, and Lemma 4.4 together playing the role of Lemma 3.1. We leave the details up to the reader. 


\section{WHITNEY DECOMPOSITION AND SWEEPING OF MEASURES}

The aim of this section is to generalize the result of [Kil2, Theorem 3.1] to the setting of metric spaces considered in this note. The proof in [Kil2] used the classical Whitney decomposition of the complement of a closed polar set. One of the primary characteristics of a Whitney decomposition is that the corresponding covering family of cubes is pairwise disjoint. In our setting we are unable to obtain this characteristic, but the decomposition we use in this section has a bounded overlap property that compensates for this shortcoming.

5.1. Definition. Let $K$ be a compact subset of $X$. A countable cover $\mathcal{F}$ of balls in $X \backslash K$ is said to be a Whitney decomposition of $X \backslash K$ if there is a constant $C>0$ so that the following three conditions hold:

(1) $\sum_{B \in \mathcal{F}} \chi_{2 B} \leq C$;

(2) $\frac{1}{2} \operatorname{rad}(B) \leq \operatorname{dist}(B, K) \leq 8 \operatorname{rad}(B)$ for every $B \in \mathcal{F}$;

(3) $2 B \subset X \backslash K$ and $\bigcup_{B \in \mathcal{F}} B=X \backslash K$.

The following proposition demonstrates the existance of such a decomposition.

5.2. Proposition. Given a compact subset $K$ of $X$, there exists a Whitney decomposition $\mathcal{F}$ of $X \backslash K$.

We refer to the proof of $\mathrm{CW}$, Theorem 1.3] on page 70 for a proof of this proposition.

We define the following Wolff potential for every compactly supported Radon measure $\nu$ on $X$.

5.3. Definition. Given a Radon measure $\nu$ on $X$, the Wolff potential of $\nu$ is the function

$$
W_{p}^{\nu}(x, r):=\int_{0}^{r}\left(t^{p} \frac{\nu(B(x, t))}{\mu(B(x, t))}\right)^{1 /(p-1)} \frac{d t}{t}
$$

for every $x \in X$ and $r>0$.

Following [Kil2, Theorem 2.15], we prove the existence of a "swept" measure of $\nu$ supported on $K$ for every finite Radon measure $\nu$ on $X$ and any compact set $K \subset X$.

5.4. Lemma. Let $K \subset X$ be a compact set and let $\nu$ be a finite Radon measure on $X$ (that is, $\nu(X)$ is finite). Then there exists a finite Radon measure $\widetilde{\nu}$, supported on $K$, so that $\nu(X) / C \leq \widetilde{\nu}(X) \leq C \nu(X)$ and for every point $x \in K$ and $r>0$,

$$
W_{p}^{\widetilde{\nu}}(x, 21 r) \geq \frac{1}{C} W_{p}^{\nu}(x, r),
$$

where $C>0$ is independent of $x$ and $r$.

Proof. Let $\mathcal{F}$ be the Whitney decomposition of $X \backslash K$ given by Proposition 5.2. For each ball $B \in \mathcal{F}$, choose a point $x_{B} \in K$ so that $d\left(x_{B}, B\right)=\operatorname{dist}(K, B)$ (this is possible because $K$ is compact). By the definition of Whitney decompositions, we see that $d\left(x_{B}, B\right) \leq 8 \operatorname{rad}(B)$. We now construct one possible measure $\widetilde{\nu}$ as follows: for sets $E \subset X$, let

$$
\widetilde{\nu}(E):=\nu(E \cap K)+\sum_{B \in \mathcal{F}} \nu(B) \delta_{x_{B}}(E),
$$


where $\delta_{x_{B}}$ is the Dirac mass supported at the point $x_{B}$. Clearly $\widetilde{\nu}$ is a Radon measure supported inside $K$, and by the bounded overlap property of $\mathcal{F}$ and by the covering property of $\mathcal{F}$, it is easily seen that

$$
\nu(X) / C \leq \widetilde{\nu}(X) \leq C \nu(X)
$$

where $C$ is the constant associated with the bounded overlap property of $\mathcal{F}$.

Let $x \in K$ and $r>0$, and consider the family $\mathcal{F}(x, r)$ to be the collection of all balls $B \in \mathcal{F}$ which intersect $B(x, r)$. If $B \in \mathcal{F}(x, r)$ and $y \in B \cap B(x, r)$, then

$$
\begin{aligned}
d\left(x_{B}, y\right) & \leq d\left(x_{B}, B\right)+2 \operatorname{rad}(B) \leq 10 \operatorname{rad}(B) \\
& \leq 20 \operatorname{dist}(B, K) \leq 20 d(x, y) \leq 20 r .
\end{aligned}
$$

Therefore, $d\left(x_{B}, x\right) \leq 21 r$, and hence

$$
\begin{aligned}
\nu(B(x, r)) & \leq \nu\left((B(x, r) \cap K) \bigcup_{B \in \mathcal{F}(x, r)} B\right) \\
& \leq \nu(B(x, r) \cap K)+\sum_{B \in \mathcal{F}(x, r)} \nu(B) \\
& \leq \nu(B(x, r) \cap K)+\sum_{x_{B} \in B(x, 21 r)} \nu(B)=\widetilde{\nu}(B(x, 21 r)) .
\end{aligned}
$$

Now from the definition of Wolff potential and by the doubling property of the measure $\mu$,

$$
\begin{aligned}
W_{p}^{\widetilde{\nu}}(x, 21 r)= & \int_{0}^{21 r}\left(t^{p} \frac{\widetilde{\nu}(B(x, t))}{\mu(B(x, t))}\right)^{1 /(p-1)} \frac{d t}{t} \\
& \geq \int_{0}^{21 r}\left(t^{p} \frac{\nu(B(x, t / 21))}{\mu(B(x, t))}\right)^{1 /(p-1)} \frac{d t}{t} \\
& \geq \frac{1}{C} \int_{0}^{r}\left(t^{p} \frac{\nu(B(x, t))}{\mu(B(x, t))}\right)^{1 /(p-1)} \frac{d t}{t}=W_{p}^{\nu}(x, r),
\end{aligned}
$$

where the constant $C$ depends on the doubling constant of $\mu$ but is indeed independent of $x$ and $r$.

\section{Finite Radon measures and $p$-Superharmonic Functions}

The $p$-superharmonic functions, defined using $p$-minimizers, are influenced by what is meant by $p$-minimizers. If, as in KinM2, KSh, Sh1, and in the first four sections of this note, we define $p$-minimizers $v$ to be functions that locally minimize the integral

$$
\int g_{w}^{p} d \mu
$$

amongst the class of all functions $w \in N_{0}^{1, p}(V)+v$, where $g_{v}$ is the minimal $p$-weak upper gradient of $v$, then the corresponding $p$-minimizers may or may not satisfy an Euler-Lagrange equation. This leads to a disadvantage in that we cannot explicitly identify the space of all finite Radon measures on $\Omega$ with the corresponding space of non-negative $p$-superharmonic functions on $\Omega$ as in HKM, KilM1, KilM2 or Mi]. To address this shortcoming, in this section we modify the definition of $p$-minimizers by using the results of [C] and [BMS]. 
In [C] Cheeger gives another definition of a Sobolev-type space, but for indices $p>1$ Cheeger's construction yields the same space as $N^{1, p}(X)$; see [Sh2, Theorem 4.10]. It is a deep theorem of Cheeger that if a metric measure space supports a doubling measure and a $(1, p)$-Poincaré inequality, then the corresponding Sobolevtype space is reflexive; see [C, Theorem 4.48]. The results of [C] can easily be extended to the case of spaces supporting a locally $Q$-regular measure and a local $(1, p)$-Poincaré inequality.

In [C, Theorem 4.38] it was shown that if the measure on $X$ is doubling and if $X$ supports a $(1, p)$-Poincaré inequality for some $p$, then there is a "differentiable" structure on $X$ compatible with the notion of an upper gradient. More precisely, the following theorem of Cheeger holds.

6.1. Theorem. Let $X$ be a metric measure space equipped with a locally doubling Borel regular measure $\mu$. Assume that $X$ admits a weak $(1, p)$-Poincaré inequality for some $1<p<\infty$. Then there exist measurable sets $U_{\alpha}$ with $\mu\left(X \backslash \bigcup_{\alpha} U_{\alpha}\right)=0$, and there exist Lipschitz "coordinate" functions

$$
\bar{X}^{\alpha}=\left(X_{1}^{\alpha}, \ldots, X_{k(\alpha)}^{\alpha}\right): X \rightarrow \mathbf{R}^{k(\alpha)}
$$

so that

(i) for each $\alpha$, the functions $X_{1}^{\alpha}, \ldots, X_{k(\alpha)}^{\alpha}$ are all linearly independent on $U_{\alpha}$ and $1 \leq k(\alpha) \leq N$, where $N$ is a constant depending only on the doubling constant of $\mu$ and the constants from the Poincaré inequality,

(ii) if $f: X \rightarrow \mathbf{R}$ is Lipschitz, then there exist unique bounded vector-valued functions $d^{\alpha} f: U_{\alpha} \rightarrow \mathbf{R}^{k(\alpha)}$ such that

$$
\lim _{r \rightarrow 0+} \sup _{x \in B\left(x_{0}, r\right)} \frac{\left|f(x)-f\left(x_{0}\right)-d^{\alpha} f\left(x_{0}\right) \cdot\left(\bar{X}^{\alpha}(x)-\bar{X}^{\alpha}\left(x_{0}\right)\right)\right|}{r}=0
$$

for $\mu$-a.e. $x_{0} \in U_{\alpha}$.

In other words, there exists a finite-dimensional $L^{\infty}$-vector bundle of Banach spaces $T^{*} X=\left\{F_{x}\right\}_{x \in X}$, the generalized cotangent bundle, and a derivation operator $D$ on the algebra of locally Lipschitz functions on $X$ taking values in the sections of $T^{*} X$. For $x$ in the "coordinate chart" $U_{\alpha}$, the fiber $F_{x}$ is the dual space to the space $\mathbf{R}^{k(\alpha)}$ mentioned in the theorem. Given a Newtonian function $f$, a norm on the space $F_{x}$ is given by

$$
\|\lambda\|_{\alpha, x}=g_{\left\langle\lambda, \bar{X}^{\alpha}\right\rangle}(x),
$$

where, for $\lambda=\left(\lambda_{1}, \ldots, \lambda_{k(\alpha)}\right)$ in $\mathbf{R}^{k(\alpha)}$, we set $\left\langle\lambda, \bar{X}^{\alpha}\right\rangle$ to be the Lipschitz function given by

$$
\sum_{i=1}^{k(\alpha)} \lambda_{i} X_{i}^{\alpha} .
$$

By linear independence of $X_{1}^{\alpha}, \ldots, X_{k(\alpha)}^{\alpha}$ we mean that whenever $g_{\left\langle\lambda, \bar{X}^{\alpha}\right\rangle}(x)=0$ for some $x \in U_{\alpha}$ we have $\lambda=0$. Finally, in a given chart $U_{\alpha}, D f$ is given by $d^{\alpha} f$.

Note that the generalized cotangent bundle is only a measurable vector bundle; that is, the charts $U_{\alpha}$ are measurable subsets of $X$ and the change of coordinate functions $\bar{X}^{\beta} \circ\left(\bar{X}^{\alpha}\right)^{-1}$ are bimeasurable invertible transformations.

By [Sh2, Theorem 4.10] and [C, Theorem 4.47], the Newtonian space $N^{1, p}(X)$ is equal to the closure in the $N^{1, p}(X)$-norm of the collection of locally Lipschitz 
functions on $X$, and by [FHK, Theorem 10], the derivation operator $D$ can be extended to all of $N^{1, p}(X)$ so that

$$
|D f(x)| \approx g_{f}(x)
$$

for all $f \in N^{1, p}(X)$ and $\mu$-a.e. $x \in X$. Here the norm $|\cdot|$ is obtained from a measurable inner product on $T^{*} X$, chosen to validate the above equivalence with $g_{f}$; see the discussion in [C].

One easily verifies (see also [C, p. 458]) that the derivation operator $D u$ satisfies the product and chain rules: if $v$ is a bounded Lipschitz function on $X, u \in N^{1, p}(X)$, and $\Phi: \mathbf{R} \rightarrow \mathbf{R}$ is continuously differentiable with bounded derivative, then $u v$ and $\Phi(u)$ belong to $N^{1, p}(X)$ and

$$
\begin{aligned}
D(u v) & =u D v+v D u, \\
D(\Phi \circ u) & =\Phi^{\prime}(u) D u .
\end{aligned}
$$

We modify the notion of $p$-harmonicity of [KinM2], Sh2], and KSh] by minimizing $|D u|$ rather than $g_{u}$.

6.2. Definition. Let $\Omega \subset X$ be an open set. A function $u: X \rightarrow[-\infty, \infty]$ is said to be $p$-harmonic on $\Omega$ if $u \in N_{\text {loc }}^{1, p}(\Omega)$ and for all relatively compact subsets $U$ of $\Omega$ and for all functions $\varphi \in N_{0}^{1, p}(U)$,

$$
\int_{U}|D u|^{p} d \mu \leq \int_{U}|D(u+\varphi)|^{p} d \mu
$$

It must be noted that by the results in $[\mathrm{C}$, the $p$-harmonic functions as defined above are $p$-quasiminimizers in the sense of $\underline{\mathrm{KSh}}$. Moreover, $p$-harmonic functions can be characterized in terms of a weak formulation of the appropriate EulerLagrange equation: $f$ is $p$-harmonic if and only if

$$
\int_{U}|D u|^{p-2} D u \cdot D \phi d \mu=0
$$

for all $U$ and $\varphi$ as in Definition 6.2. Here we make use of the fact that the norms $|\cdot|$ on the fibers of $T^{*} X$ can be chosen to be inner product norms; see the discussion in [C, p. 460].

6.3. Definition. A function $u \in N_{\mathrm{loc}}^{1, p}(\Omega)$ is said to be a $p$-supersolution if for all relatively compact open subsets $V$ of $\Omega$ and for all functions $\varphi \in N_{0}^{1, p}(V)$ so that $\varphi \geq 0$

or equivalently,

$$
\int_{V}|D u|^{p-2} D u \cdot D \varphi d \mu \geq 0
$$

$$
\int_{V}|D u|^{p} d \mu \leq \int_{V}|D(u+\varphi)|^{p} d \mu .
$$

Remarks. (i) A function is $p$-harmonic if and only if it is both a $p$-supersolution and a $p$-subsolution (a function $u$ is a $p$-subsolution if $-u$ is a $p$-supersolution). For the remainder of this section we consider the $p$-superharmonic functions obtained from Definition 1.12 by using $p$-harmonic functions instead of $p$-minimizers in the comparison tests. We also define solutions to obstacle problems by minimizing the integral $\int|D u|^{p} d \mu$ rather than $\int g_{u}^{p} d \mu$.

(ii) Since $p$-harmonic functions and $p$-supersolutions exhibit the minimizing property of $p$-minimizers and $p$-superminimizers respectively, the results of the first three 
sections of this note as well as the results of [Sh1, KinM2, and KSh] apply to the modified versions of $p$-superharmonic functions.

(iii) The above definition of $p$-harmonic functions and $p$-supersolutions has been studied in BMS using the techniques developed in KilM1, KilM2 and Mi], and it has been shown in BMS that the set of all non-negative $p$-supersolutions $u \in N_{0}^{1, p}(\Omega)$ and the Radon measures $\nu$ in the positive cone in the dual space $N_{0}^{1, p}(\Omega)^{*}$ are in one-to-one correspondence given by

$$
\int_{\Omega}|D u|^{p-2} D u \cdot D \varphi d \mu=\int_{\Omega} \varphi d \nu
$$

whenever $\varphi \in N_{0}^{1, p}(\Omega)$.

We extend the results of $\mathrm{BMS}$ ] and construct a correspondence between compactly supported finite Radon measures on $\Omega$ and non-negative $p$-superharmonic functions with vanishing boundary values, thus extending the results of [KilM1], KilM2 and Mi to the metric space setting. We will use this correspondence together with the sweeping technique of the previous section to construct a $p$ superharmonic function on $\Omega$ whose infinity set is precisely the given zero capacity set.

We first show that given a $p$-superharmonic function, there is an associated finite Radon measure $\nu$ supported on $\Omega$, related to $u$ via equation (6.4) above. It was shown in [KinM2, Corollary 7.8] that if $k$ is a positive integer, then

$$
u_{k}=\min \{u, k\}
$$

is in $N_{\text {loc }}^{1, p}(\Omega)$, and hence $D u_{k}$ is well defined a.e., and moreover, if $j>k$, then $D u_{j}=D u_{k}$ on the set

$$
\{x \in \Omega: u(x) \leq k\} .
$$

6.5. Definition. Let $u$ be a non-negative $p$-superharmonic function on $\Omega$. Then

$$
D u:=\lim _{k \rightarrow \infty} D u_{k}
$$

is well defined a.e. on the set $\{x \in \Omega: u(x)<\infty\}$.

The arguments in [HKM, Theorem 7.46] and [KilM1, Theorem 1.15] show that in the Euclidean setting with $D u=\nabla u,|D u| \in L^{1}(\Omega)$. Their argument is quite general, and is easily adapted to our situation; see below.

6.6. Lemma. Let $\Omega$ be a bounded domain with $\operatorname{cap}_{p}(X \backslash \Omega)>0$ and let $u$ be a non-negative $p$-superharmonic function on $\Omega$. Then $|D u| \in L_{\mathrm{loc}}^{p-1}(\Omega)$.

Proof. By [KinM2, Corollary 7.8] the function

$$
u_{k}=\min \{u, k\}
$$

is a $p$-supersolution and $u_{k} \in N_{\text {loc }}^{1, p}(\Omega)$. By the Caccioppoli-type inequality BMS, Lemma 4.4], if $B$ is a ball in $X$ so that $3 B \subset \Omega$ and $\eta \geq 0$ is a Lipschitz function with support in $2 B$ so that $\eta=1$ on $B$, then

$$
\begin{aligned}
\int_{B}\left|D u_{k}\right|^{p} u_{k}^{-(1+\epsilon)} d \mu & \leq \int_{2 B}\left|D u_{k}\right|^{p} \eta^{p} u_{k}^{-(1+\epsilon)} d \mu \\
& \leq C \int_{2 B} u_{k}^{p-(1+\epsilon)}|D \eta|^{p} d \mu \leq C_{B} \int_{2 B} u_{k}^{p-(1+\epsilon)} d \mu .
\end{aligned}
$$


Let $B$ be a ball in $X$ so that $5 B \subset \Omega$. By [BMS, Lemma 4.5] and by the fact that $X$ supports a $\left(p_{0}, p\right)$-Poincaré inequality for some $p_{0}>p$ (see [HaK, Section 5]), we have that

$$
\left(f_{B} u^{\gamma} d \mu\right)^{1 / \gamma} \leq C_{\gamma} \inf _{2 B} u
$$

whenever $\gamma<p_{0}(p-1) / p$. Here the constant $C_{\gamma}$ is independent of $u$ and $B$, but may depend on $\gamma$. Just as in the alternative proof of [HKM, Theorem 7.46], if $\epsilon>0$, then

$$
\begin{aligned}
\int_{B}\left|D u_{k}\right|^{p-1} d \mu & =\int_{B}\left|D u_{k}\right|^{p-1} u_{k}^{-(1+\epsilon)(p-1) / p} u_{k}^{(1+\epsilon)(p-1) / p} d \mu \\
& \leq\left(\int_{B}\left|D u_{k}\right|^{p} u_{k}^{-(1+\epsilon)} d \mu\right)^{(p-1) / p}\left(\int_{B} u_{k}^{(1+\epsilon)(p-1)} d \mu\right)^{1 / p} \\
& \leq\left(\int_{B}\left|D u_{k}\right|^{p} u_{k}^{-(1+\epsilon)} d \mu\right)^{(p-1) / p}\left(\int_{B} u^{(1+\epsilon)(p-1)} d \mu\right)^{1 / p} \\
& \leq C_{B}\left(\int_{2 B} u^{p-(1+\epsilon)} d \mu\right)^{(p-1) / p}\left(\int_{B} u^{(1+\epsilon)(p-1)} d \mu\right)^{1 / p} .
\end{aligned}
$$

Since $p_{0} / p>1$, we can find $\epsilon>0$ so that $1+\epsilon<p_{0} / p$; that is, $(1+\epsilon)(p-1)<$ $p_{0}(p-1) / p$. Thus by Proposition 2.2 and inequality (6.7) above,

$$
\int_{B}\left|D u_{k}\right|^{p-1} d \mu \leq C_{B, p, p_{0}}\left(\inf _{B} u\right)^{p-1}<\infty .
$$

Therefore the sequence $\left|D u_{k}\right|$ is a bounded sequence in $L_{\text {loc }}^{p-1}(\Omega)$, and hence by the reflexivity of $L_{\text {loc }}^{p-1}(\Omega)$ we conclude that $|D u| \in L_{\text {loc }}^{p-1}(\Omega)$.

The next proposition is a metric space analog of [KilM1, Theorem 1.15], and associates to each non-negative $p$-superharmonic function a Radon measure $\nu$.

6.8. Proposition. If $u$ is a non-negative p-superharmonic function on domain $\Omega$, then there is a Radon measure $\nu$, supported on $\bar{\Omega}$, so that for every $\varphi \in \operatorname{Lip}_{c}(\Omega)$,

$$
\int_{\Omega}|D u|^{p-2} D u \cdot D \varphi d \mu=\int_{\Omega} \varphi d \nu
$$

Observe that the equation given in the above proposition is precisely equation (6.4).

Proof. Let $u_{k}=\min \{u, k\}$, and let $\varphi \in \operatorname{Lip}_{c}(\Omega)$. Since $u_{k} \in N_{\text {loc }}^{1, p}(\Omega)$ and $\varphi$ has compact support in $\Omega$, by [BMS, Proposition 3.5] there is a Radon measure $\nu_{k}$ supported in $\bar{\Omega}$ so that

$$
\int_{\Omega}\left|D u_{k}\right|^{p-2} D u_{k} \cdot D \varphi d \mu=\int_{\Omega} \varphi d \nu_{k} .
$$

By Lemma 6.6 above, $\left|D u_{k}\right|^{p-2} D u_{k} \cdot D \varphi$ converges to $|D u|^{p-2} D u \cdot D \varphi$ in $L^{1}(\Omega)$. Therefore, $\left(\nu_{k}\right)_{k}$ is a bounded sequence of Radon measures on compact subsets of $\Omega$, and hence weakly converge to a Radon measure $\nu$; that is,

$$
\int_{\Omega} \varphi d \nu_{k} \rightarrow \int_{\Omega} \varphi d \nu
$$


for every $\varphi \in \operatorname{Lip}_{c}(\Omega)$. Thus by the convergence of $\left|D u_{k}\right|^{p-2} D u_{k} \cdot D \varphi$ to $|D u|^{p-2} D u$. $D \varphi$ again, we see that

$$
\int_{\Omega}|D u|^{p-2} D u \cdot D \varphi d \mu=\int_{\Omega} \varphi d \nu
$$

Remark. Unlike in BMS, Proposition 3.5], we cannot replace the condition of $\varphi \in$ $\operatorname{Lip}_{c}(\Omega)$ with $\varphi \in N_{0}^{1, p}(\Omega)$ because unlike the Radon measures associated with $p$ supersolutions, the Radon measures associated to $p$-superharmonic functions as in the above proposition may charge sets of zero $p$-capacity.

The aim of the next section is to construct a $p$-superharmonic function whose infinity set is precisely the closed zero $p$-capacity set $E$ under study; this would be a refinement of Theorem 3.4. In order to construct such a $p$-superharmonic function we follow the lead of Kil2. We sweep the Radon measure associated with the $p$-superharmonic function constructed in Theorem 3.4 onto the set $E$ using the technique developed in Section 5, and use the swept measure to construct the desired $p$-superharmonic function. The rest of this section is devoted to showing that for any finite Radon measure there is a corresponding $p$-harmonic function related to it as in (6.4). The argument closely follows the proofs of KilM1, Theorem 2.4] and [Mi, Theorem 2.21] and uses the machinery set up in [BMS].

6.9. Proposition. Given a finite Radon measure $\nu$ on the domain $\Omega$, there exists a non-negative $p$-superharmonic function $u$ on $\Omega$ so that for all $\varphi \in \operatorname{Lip}_{c}(\Omega)$,

$$
\int_{\Omega}|D u|^{p-2} D u \cdot D \varphi d \mu=\int_{\Omega} \varphi d \nu .
$$

Proof. By BMS, Lemma 3.12] there exists a sequence $\left(\nu_{k}\right)_{k}$ of Radon measures on $\Omega$ so that $\nu_{k} \rightarrow \nu$ weakly and $\nu_{k}(\Omega) \leq \nu(\Omega)$, and by [BMS, Proposition 3.9] there is an associated sequence of $p$-supersolutions $u_{k} \in N_{0}^{1, p}(\Omega)$ so that for every $k$,

$$
\int_{\Omega}\left|D u_{k}\right|^{p-2} D u_{k} \cdot D \varphi d \mu=\int_{\Omega} \varphi d \nu_{k}
$$

Note that

$$
\int_{\Omega} \varphi d \nu_{k} \rightarrow \int_{\Omega} \varphi d \nu
$$

as $k \rightarrow \infty$. For positive integers $j$ set

$$
u_{k, j}=\min \left\{u_{k}, j\right\} .
$$

Then as $\left|D u_{k, j}\right|=0$ on the set where $u_{k} \geq j$ and $u_{k, j} \in N_{0}^{1, p}(\Omega)$, we see that

$$
\begin{aligned}
\int_{\Omega}\left|D u_{k, j}\right|^{p} d \mu & =\int_{\Omega}\left|D u_{k j}\right|^{p-2} D u_{k, j} \cdot D u_{k, j} d \mu \\
& =\int_{\Omega}\left|D u_{k}\right|^{p-2} D u_{k} \cdot D u_{k, j} d \mu \\
& =\int_{\Omega} u_{k, j} d \nu_{k} \leq j \nu_{k}(\Omega) \leq j \nu(\Omega) .
\end{aligned}
$$

Therefore, by the Sobolev inequality [KSh, Lemma 2.10], we have

$$
\int_{\Omega} u_{k, j}^{p} d \mu \leq C \int_{\Omega}\left|D u_{k, j}\right|^{p} d \mu \leq C \nu(\Omega) j .
$$


Now,

$$
k^{p} \mu\left(\left\{x \in \Omega: j \leq u_{k}(x)<2 j\right\}\right) \leq \int_{\Omega} u_{k, 2 j}^{p} d \mu \leq 2 C j .
$$

Thus, by Hölder's inequality, when $0<q<1$,

$$
\begin{aligned}
& \int_{\left\{j \leq u_{k}<2 j\right\}}\left|D u_{k, 2 j}\right|^{q(p-1)} d \mu \\
& \leq \mu\left(\left\{j \leq u_{k}<2 j\right\}\right)^{1-q(p-1) / p}\left(\int_{\Omega}\left|D u_{k, 2 j}\right|^{p} d \mu\right)^{q(p-1) / p} \leq C j^{-(1-q)(p-1)} .
\end{aligned}
$$

Note that $1-q>0$. Hence, when $0<i<2^{m+1}$,

$$
\begin{aligned}
\int_{\Omega}\left|D u_{k, i}\right|^{q(p-1)} d \mu & \leq \sum_{j=0}^{m} \int_{\left\{2^{j}<u_{k} \leq 2^{j+1}\right\}}\left|D u_{k, 2^{j}}\right|^{q(p-1)} d \mu \\
& \leq C \sum_{j=0}^{m} 2^{-j(1-q)(p-1)} \leq C(p, q) .
\end{aligned}
$$

Therefore, as $u_{k, i} \rightarrow u_{k}$ in $N_{0}^{1, p}(\Omega)$, we have

$$
\int_{\Omega}\left|D u_{k}\right|^{q(p-1)} d \mu \leq C(p, q)
$$

the bound being independent of $k$. Hence without loss of generality we can assume that the sequence $\left|D u_{k}\right|^{p-2} D u_{k}$ is a weakly convergent sequence in $L^{1}\left(\Omega ; \mathbf{R}^{N}\right)$.

For every positive integer $j$ the sequence $\left(u_{k, j}\right)_{k}$ is bounded in $N_{0}^{1, p}(\Omega)$. By the reflexivity of $N_{0}^{1, p}(\Omega)$ we can assume that for every positive integer $j$ the sequence $\left(u_{k, j}\right)_{k}$ converges weakly to a function $v_{j}$ in $N_{0}^{1, p}(\Omega)$ and

$$
\left|D u_{k, j}\right|^{p-2} D u_{k, j} \rightarrow\left|D v_{j}\right|^{p-2} D v_{j}
$$

weakly in $L^{1}\left(\Omega ; \mathbf{R}^{N}\right)$.

Moreover, we claim that $v_{j} \leq v_{j+1}$. To see this, we use a careful diagonalization argument. Since $\left(u_{k, j}\right)_{k}$ converges weakly to $v_{j}$, by Mazur's lemma we can choose a convex combination of this sequence converging strongly to $v_{j}$ in $N_{0}^{1, p}(\Omega)$. Consider the corresponding convex combination sequence obtained from $\left(u_{k, j+1}\right)_{j}$; it converges weakly to $v_{j+1}$ as well. Now obtain a corresponding convex combination of this new sequence converging strongly to $v_{j+1}$. Since $u_{k, j} \leq u_{k, j+1}$, the convex combination of the convex combination that gave $v_{j+1}$ has every term larger than the corresponding convex combination of convex combination of $\left(u_{k, j}\right)_{k}$ as well, which again converges strongly to $v_{j}$. Hence $v_{j} \leq v_{j+1}$.

Since

$$
v_{j}=\lim _{i \rightarrow \infty} \inf _{k \geq i} u_{k, j}=\lim _{i \rightarrow \infty} \lim _{l \rightarrow \infty} \inf _{i \leq k \leq l} u_{k, j}
$$

is $p$-superharmonic on $\Omega$ and $v_{j} \leq j$, by [KinM2, Corollary 7.9] we conclude that $v_{j}$ is a $p$-supersolution on $\Omega$. Hence by [KinM2, Lemma 7.1] we see that the lower semicontinuous regularization of the function $v=\lim _{j \rightarrow \infty} v_{j}$ is either a $p$-superharmonic function on $\Omega$ or is identically infinite on $\Omega$.

Suppose the lower semicontinuous regularization of $v$ is identically infinite. Then $v$ is infinite a.e.; that is, $v_{j}(x) \rightarrow \infty$ for a.e. $x \in \Omega$. Since

$$
v_{j}=\liminf _{k \rightarrow \infty} u_{k, j}
$$


this implies that $v_{j}=j$ a.e. on $\Omega$. This contradicts the fact that $v_{j} \in N_{0}^{1, p}(\Omega)$ because of the $(1, p)$-Poincaré inequality. Thus $v$ is $p$-superharmonic on $\Omega$, and hence by Proposition $2.2 v$ is finite $p$-q.e. in $\Omega$. Consequently,

$$
v=\liminf _{k \rightarrow \infty} u_{k}
$$

on the set where $v$ is finite. Moreover, as $\min \{v, j\}=v_{j}$, we see by the definition of $D v$ and by the proof of Lemma 6.6 that

$$
\left|D v_{j}\right|^{p-2} D v_{j} \rightarrow|D v|^{p-2} D v
$$

weakly in $L^{1}\left(\Omega ; \mathbf{R}^{N}\right)$. The weak convergence of $D u_{k, j}$ to $D v_{j}$ now indicates that

$$
\int_{\Omega}\left|D u_{k, j}\right|^{p-2} D u_{k, j} \cdot D \varphi d \mu \rightarrow \int_{\Omega}|D v|^{p-2} D v \cdot D \varphi d \mu
$$

as $k$ and then $j$ tend to infinity. Since $D u_{k, j}=D u_{k}$ wherever $u_{k} \leq j$ (almost everywhere), and $D u_{k, j}=0$ wherever $u_{k}>j$, we see by the weak convergence of $\left|D u_{k}\right|^{p-2} D u_{k}$ that

$$
\int_{\Omega}\left|D u_{k}\right|^{p-2} D u_{k} \cdot D \varphi d \mu \rightarrow \int_{\Omega}|D v|^{p-2} D v \cdot D \varphi d \mu
$$

and hence

$$
\int_{\Omega}|D v|^{p-2} D v \cdot D \varphi d \mu=\int_{\Omega} \varphi d \nu
$$

The lower semicontinuous regularization $u$ of $v$ now satisfies the conditions of the proposition.

\section{COMPACT ZERO $p$-CAPACITY SETS AND $\infty$-SETS OF MODIFIED $p$-SUPERHARMONIC FUNCTIONS}

In the previous section we studied the relations of the $p$-superharmonic functions to the Radon measures. The next lemma is an adaptation of [KilM2, Theorem 1.6] (see also [Mi, Theorem 3.1]) to the metric setting.

7.1. Lemma. Let $u$ be a non-negative p-superharmonic function on $\Omega$ in the sense of section 6 and let $\nu$ be the measure associated with $u$ as in Propositions 6.8 and 6.9. Then there is a constant $C>0$ so that for every ball $B=B(x, r)$ with $4 B \subset \Omega$,

$$
\frac{1}{C} W_{p}^{\nu}(x, r) \leq u(x) \leq C\left(\inf _{B} u+W_{p}^{\nu}(x, 2 r)\right) .
$$

The proof of this lemma follows the same lines as the proofs of BMS, Proposition 4.9] and [BMS, Proposition 4.10], once [BMS, Lemma 4.8] is extended to $p$-superharmonic functions; this can be done just as in [KilM1, Lemma 3.5] and [Mi, Lemma 3.5], and is left to the reader to verify.

Now we are ready to state and prove the main result of this section.

7.2. Theorem. Let $E$ be a compact subset of $\Omega$ so that $\operatorname{cap}_{p}(E)=0$. Then there exists a non-negative p-superharmonic function $u$ on $\Omega$ so that

$$
E=\{x \in \Omega: u(x)=\infty\} .
$$

Proof. By Theorem 3.4 there exists a non-negative $p$-superharmonic function $v$ on $\Omega$ so that $E \subset\{x \in \Omega: v(x)=\infty\}$. Let $\nu$ be the Radon measure associated with $v$ as in Proposition 6.8. Let $\widetilde{\nu}$ be the swept measure corresponding to $\nu$ constructed via 
Lemma 5.4, and let $u$ be the non-negative $p$-superharmonic function on $\Omega$ associated with $\widetilde{\nu}$ as in Proposition 6.9. We show that $u$ satisfies the above theorem.

Since $v=\infty$ on $E$, by Lemma 7.1 we see that $W_{p}^{\nu}(x, r)=\infty$ for all $x \in E$ and for all $r>0$ so that $B(x, 4 r) \subset \Omega$. Hence by Lemma $5.4, W_{p}^{\widetilde{\nu}}(x, r)=\infty$ whenever $x \in E$ and $B(x, 84 r) \subset \Omega$. Again by Lemma 7.1, we have $u(x)=\infty$ when $x \in E$.

On the other hand, since $\widetilde{\nu}$ is supported on $E$ and $E$ is compact, if $x \in \Omega \backslash E$ there is a radius $r>0$ so that $W_{p}^{\widetilde{\nu}}(x, 2 r)=0$, and hence by Lemma 7.1 and Proposition 2.2 we see that $u(x)$ is finite. Thus

$$
E=\{x \in \Omega: u(x)=\infty\} .
$$

\section{REFERENCES}

[B] J. Björn, Boundary continuity for quasi-minimizers on metric spaces, Illinois J. Math. 46 (2002), 383-403. MR.1936925 (2003i:49068)

[BMS] J. Björn, P. MacManus, and N. Shanmugalingam, Fat sets and pointwise boundary estimates for p-harmonic functions in metric spaces, J. Anal. Math. 85 (2001), 339-369. MR 1869615 (2002j:31017)

[C] J. Cheeger, Differentiability of Lipschitz functions on metric measure spaces, Geom. Funct. Anal. 9 (1999), 428-517. MR.1708448 (2000g:53043)

[CW] R. R. Coifman and G. Weiss, Analyse harmonique non-commutative sur certains espaces homogènes, Lecture Notes in Mathematics, Springer-Verlag, Berlin, 1971. MR0499948 (58:17690)

[FHK] B. Franchi, P. Hajłasz, and P. Koskela, Definitions of Sobolev classes on metric spaces, Ann. Inst. Fourier (Grenoble) 49 (1999), 1903-1924. MR.1738070 (2001a:46033)

[HaK] P. Hajłasz and P. Koskela, Sobolev met Poincaré, Mem. Amer. Math. Soc. 145 (2000). MR.1683160 (2000j:46063)

[He] J. Heinonen, Lectures on Analysis on Metric Spaces, Springer-Verlag, New York, 2001. MR.1800917 (2002c:30028)

[HKM] J. Heinonen, T. Kilpeläinen, and O. Martio, Nonlinear potential theory of degenerate elliptic equations, Oxford Science Publications, Clarendon Press, Oxford, 1993. MR:1207810 (94e:31003)

[Ho] I. Holopainen, Nonlinear potential theory and quasiregular mappings on Riemannian manifolds, Ann. Acad. Sci. Fenn. Ser. A I Math. Dissertationes 74 (1990). MR1052971 (91e:31029)

[KaS] S. Kallunki and N. Shanmugalingam, Modulus and continuous capacity, Ann. Acad. Sci. Fenn. Math. 26 (2001), 455-464. MR1833251(2002c:31008)

[Kil1] T. Kilpeläinen, Potential theory for supersolutions of degenerate elliptic equations, Indiana Univ. Math. J. 38 (1989), 253-275. MR0997383 (90e:35061)

[Kil2] T. Kilpeläinen, Singular solutions to p-Laplacian type equations, Ark. Mat. 37 (1999), 275-289. MR.1714768 (2000k:31010)

$[\mathrm{KKM}]$ T. Kilpeläinen, J. Kinnunen and O. Martio, Sobolev spaces with zero boundary values on metric spaces, Potential Anal. 12 (2000), 233-247. MR.1752853 (2000m:46071)

[KilM1] T. Kilpeläinen and J. Malý, Degenerate elliptic equations with measure data and nonlinear potentials, Ann. Scuola Norm. Sup. Pisa Cl. Sci. 19 (1992), 591-613. MR1205885 (94c:35091)

[KilM2] T. Kilpeläinen and J. Malý, The Wiener test and potential estimates for quasilinear elliptic equations, Acta Math. 172 (1994), 137-161. MR1264000(95a:35050)

[KinM1] J. Kinnunen and O. Martio, The Sobolev capacity on metric spaces, Ann. Acad. Sci. Fenn. Math. 21 (1996), 367-382. MR.1404091 (98c:46063)

[KinM2] J. Kinnunen and O. Martio, Nonlinear potential theory on metric spaces, Illinois J. Math. 46 (2002), 857-883. MR1951245(2005e:31013)

[KSh] J. Kinnunen and N. Shanmugalingam, Regularity of quasi-minimizers on metric spaces, Manuscripta Math. 105 (2001), 401-423. MR1856619 (2002i:35054) 
[KoM] P. Koskela and P. MacManus, Quasiconformal mappings and Sobolev spaces, Studia Math. 131 (1998), 1-17. MR1628655 (99e:46042)

[KST] P. Koskela, N. Shanmugalingam, and H. Tuominen, Removable sets for the Poincaré inequality on metric spaces, Indiana Univ. Math. J. 49 (2000), 333-352. MR1777027 (2001g:46076)

[Mi] P. Mikkonen, On the Wolff potential and quasilinear elliptic equations involving measures, Ann. Acad. Sci. Fenn. Math. Diss. 104 (1996). MR.1386213 (97e:35069)

[Sh1] N. Shanmugalingam, Harmonic functions on metric spaces, Illinois J. Math. 45 (2001), 1021-1050. MR.1879250 (2003c:31010)

[Sh2] N. Shanmugalingam, Newtonian spaces: An extension of Sobolev spaces to metric measure spaces, Rev. Mat. Iberoamericana 16 (2000), 243-279. MR1809341(2002b:46059)

[Sh3] N. Shanmugalingam, Some convergence results for $p$-harmonic functions on metric measure spaces, Proc. London Math. Soc. 83 (2003), 226-246. MR.1978575 (2005f:31010)

Department of Mathematical Sciences, P.O. Box 3000, FI-90014 University of Oulu, FinLAND

E-mail address: juha.kinnunen@oulu.fi

Department of Mathematical Sciences, P.O. Box 210025, University of Cincinnati, Cincinnati, Ohio 45221-0025

E-mail address: nages@math.uc.edu 